Supporting Information

\title{
Electrical Detection of Molecular Transformations Associated with Chemical Reactions Using Graphene Devices
}

Yuri Sakamoto ${ }^{1}$, Takashi Ikuta ${ }^{* 2}$ and Kenzo Maehashi ${ }^{* 2}$

1) Graduate school of Bio-Applications and Systems Engineering, Tokyo University of

Agriculture and Technology, 2-24-16, Nakacho, Koganei, Tokyo 184-8588, Japan

2) Division of Advanced Applied Physics, Institute of Engineering, Tokyo University of Agriculture and Technology, 2-24-16, Nakacho, Koganei, Tokyo 184-8588, Japan

Corresponding Author

*Takashi Ikuta: Email ikuta@go.tuat.ac.jp

*Kenzo Maehashi: Email maehashi@,cc.tuat.ac.jp 
S1. Evaluating the graphene properties before and after the FET-fabrication process by Raman spectroscopy

The Raman spectroscopy measurements were performed on the pristine graphene before and after the lithography process. The intensities of the Raman spectra were normalized by the G peak intensity. The intensity ratios of the Raman spectrum before etching was estimated to be $\mathrm{G} / \mathrm{G}^{\prime}=$ 2.27 and $\mathrm{D} / \mathrm{G}=0.04$, indicating that the graphene is a monolayer with few defects. The Raman spectrum after the FET-fabrication process has $\mathrm{G} / \mathrm{G}^{\prime}=2.10$ and $\mathrm{D} / \mathrm{G}=0.13$, indicating that the monolayer graphene remained clean after etching by covering the graphene channel with photoresist and irradiating the rest of the graphene with oxygen plasma.

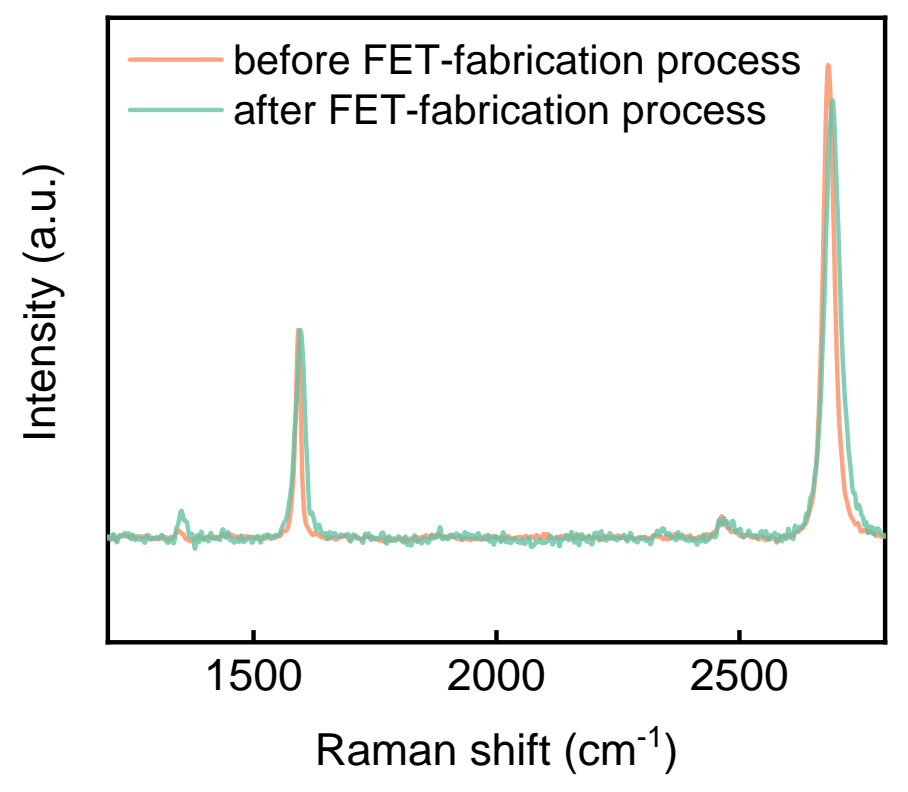

Figure S1. Raman spectra of the pristine graphene before (green) and after (orange) the FETfabrication process. 
$\mathrm{S} 2$. Evaluating the graphene properties before and after the FET-fabrication process by Atomic Force Microscopy

Atomic Force Microscopy (AFM) performed on the graphene before and after the FETfabrication process. The AFM results showed that the root mean square roughness Rq, which indicates the standard deviation of surface roughness, was estimated to be 0.226 and $0.245 \mathrm{~nm}$ for graphene before and after fabrication, respectively. The value of $\mathrm{Rq}$ for $\mathrm{SiO}_{2} / \mathrm{Si}$ substrate was $0.188 \mathrm{~nm}$, and the change in roughness before and after fabrication was sufficiently small, which suggests that there was almost no residue on the channel surface due to the FET-fabrication process.

\section{(a)}

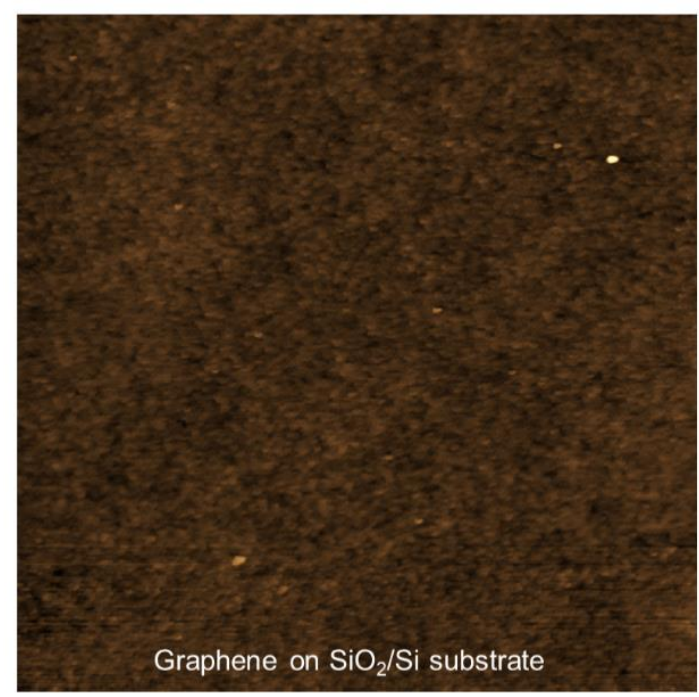

$1.00 \mu \mathrm{m}$ (b)

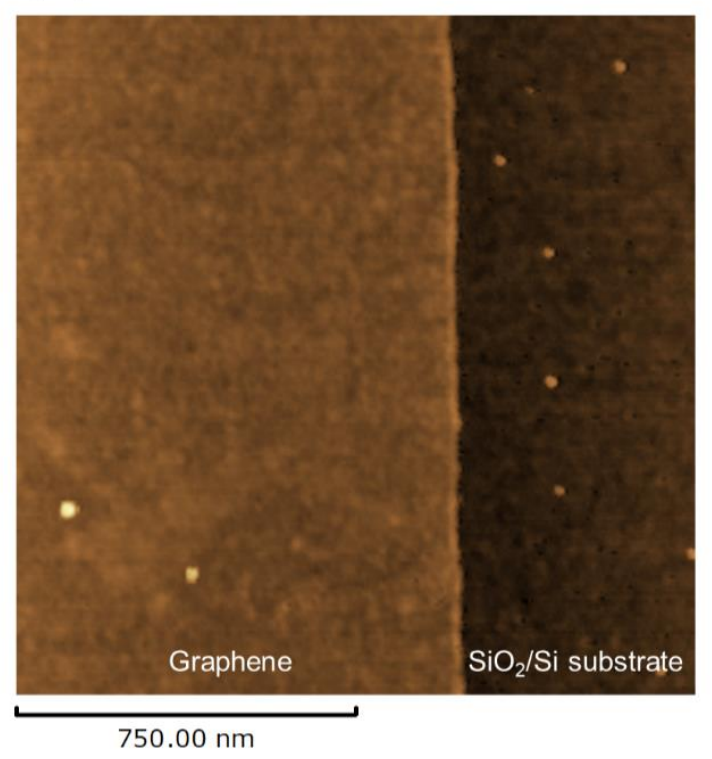

Figure S2. AFM images of the graphene on $\mathrm{SiO}_{2} / \mathrm{Si}$ (a) before and (b) after the FET-fabrication process. 
S3. Evaluating the hysteresis effect of the back-gate voltage sweep on a graphene FET

The transfer characteristic of the pristine graphene FET were measured by applying the backgate voltage back and forth. The results show that there is almost no hysteresis. For reference, the Dirac-point voltage shift of back and forth was less than $0.1 \mathrm{~V}$.

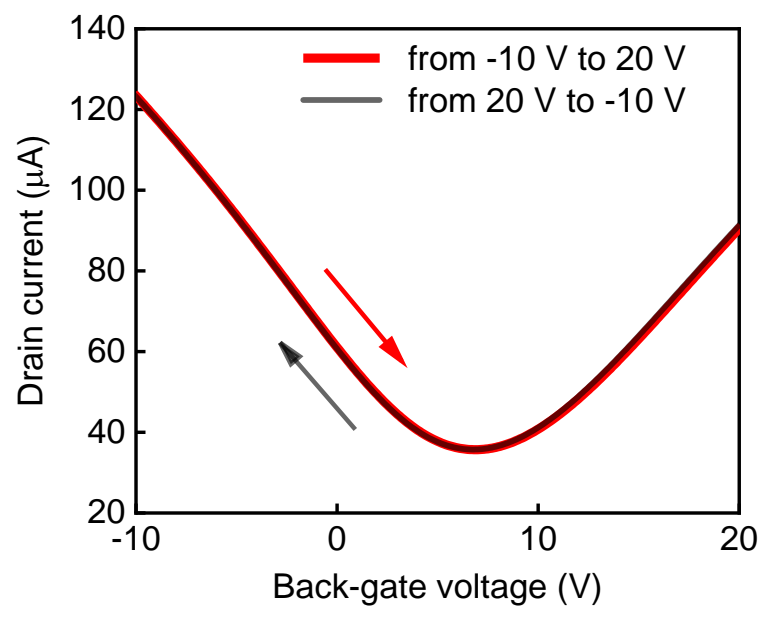

Figure S3. Transfer characteristics of the pristine graphene FET. The back-gate voltage changed from -10 to 20 (red) $\mathrm{V}$ and from 20 to $-10 \mathrm{~V}$ (black), while applying a drain-source voltage of 50 $\mathrm{mV}$. 
S4. Determining the density of modified molecules on graphene with a CV measurement

CV measurements were performed to evaluate the modification density of NAM on graphene. $\mathrm{Ti}$ and $\mathrm{Au}$ were deposited on the $\mathrm{SiO}_{2} / \mathrm{Si}$ substrates as electrodes for contact, and then the synthesized graphene was transferred onto the substrates. Then, they were immersed in $1 \mathrm{mM}$ of 11-(Ferrocenyl)undecanethiol solution under UV-irradiation for $3 \mathrm{~h}$. The $\mathrm{CV}$ measurement cell for a plate sample (BAS Inc., Plate Material Evaluating Cell) was used with the fabricated sample as the working electrode, the $\mathrm{Ag} / \mathrm{AgCl}$ electrode served as the reference electrode, and $\mathrm{Pt}$ was used as the counter electrode. The voltametric responses were recorded in acetonitrile that contained 1 $\mathrm{mM}$ of tetrabutylammonium tetrafluoroborate by using a source measurement unit (Keysight, B2912A). ${ }^{1-3}$. The scanning rate was $75 \mathrm{mV} \mathrm{s}^{-1}$. The obtained voltammograms were integrated and the modified density was calculated while considering the area of the sample.

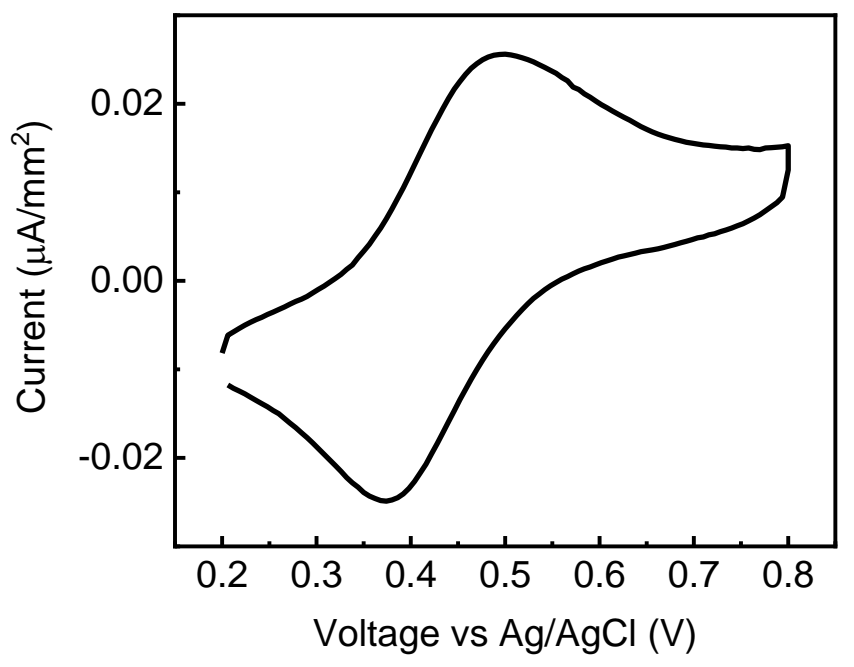

Figure S4. CV traces of the NAM modified graphene on the $\mathrm{SiO}_{2} / \mathrm{Si}$ substrate after it reacted with 11-(Ferrocenyl)undecanethiol. 
S5. Evaluating the change in transfer characteristics of the pristine graphene FET before and after the UV irradiation

The results show that there is almost no shift in the transfer characteristics before and after the UV irradiation. For reference, the difference in the Dirac-point voltage before and after UV irradiation was less than $0.05 \mathrm{~V}$.

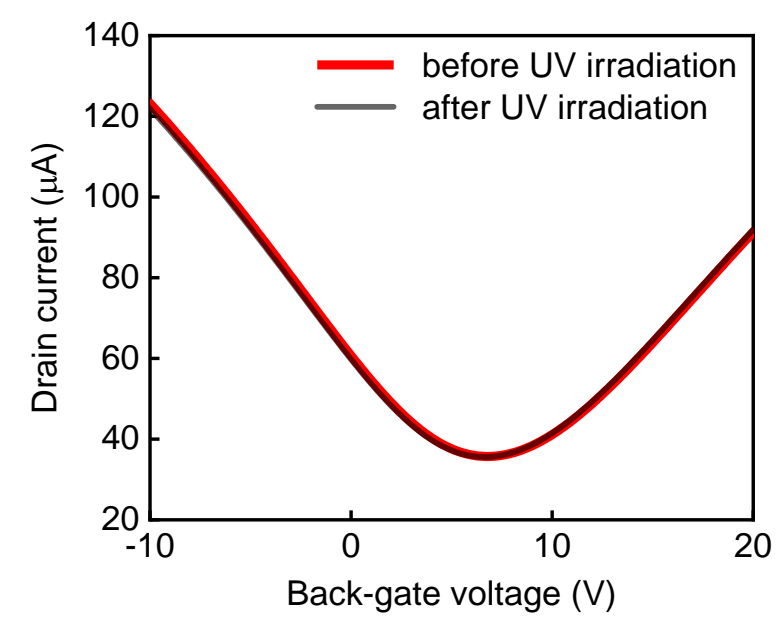

Figure S5. Transfer characteristics of the pristine graphene FET before (red) and after (black) the UV irradiation. 
S6. Transfer characteristics changes for each concentrations of MeSH

Time evolutions of the mobility and Dirac-point voltage shift was measured with MeSH for the concentrations of 10,50 , and $100 \mathrm{ppb}$. The time constant did not change much for the three different concentrations, suggesting that MeSH was in excess for the NAM reaction site on graphene at these concentrations.

(a)

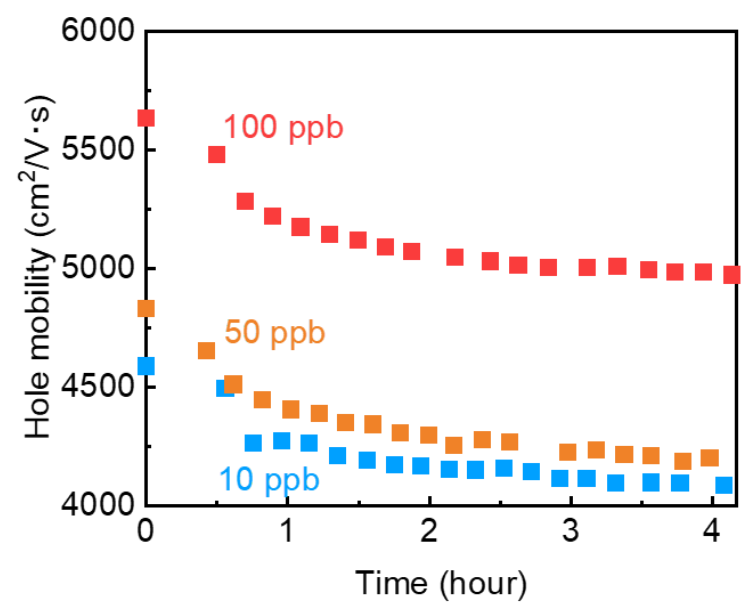

(b)

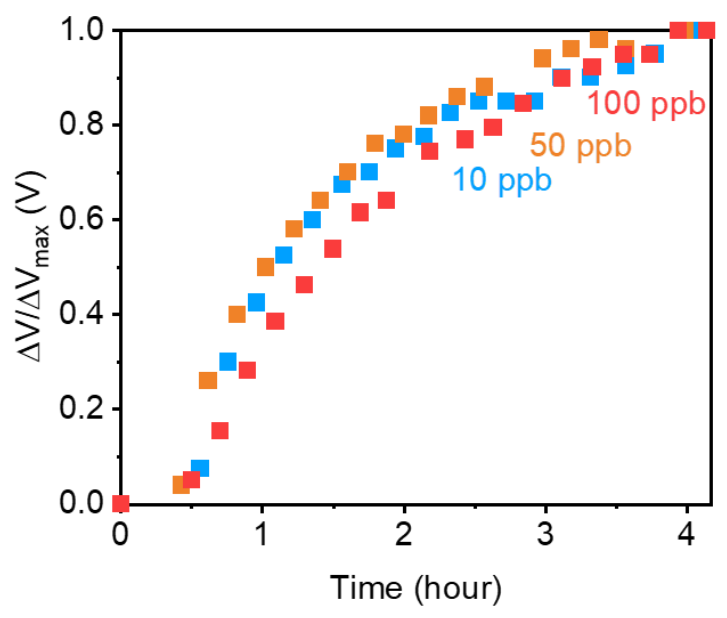

Figure S6. Time evolution of the (a) mobility and (b) Dirac-point voltage shift with MeSH for the concentrations of 10 (blue), 50 (orange), and 100 (red) ppb. 
S7. Reproducibility of mobility change

The transfer characteristics were measured several times under the same condition for the different devices. The time evolution of the hole and electron mobility during the reaction with MeSH under UV irradiation showed a similar tendency. In addition, these results also gave comparable b-values, $\mathrm{b}=1.4$ for both.
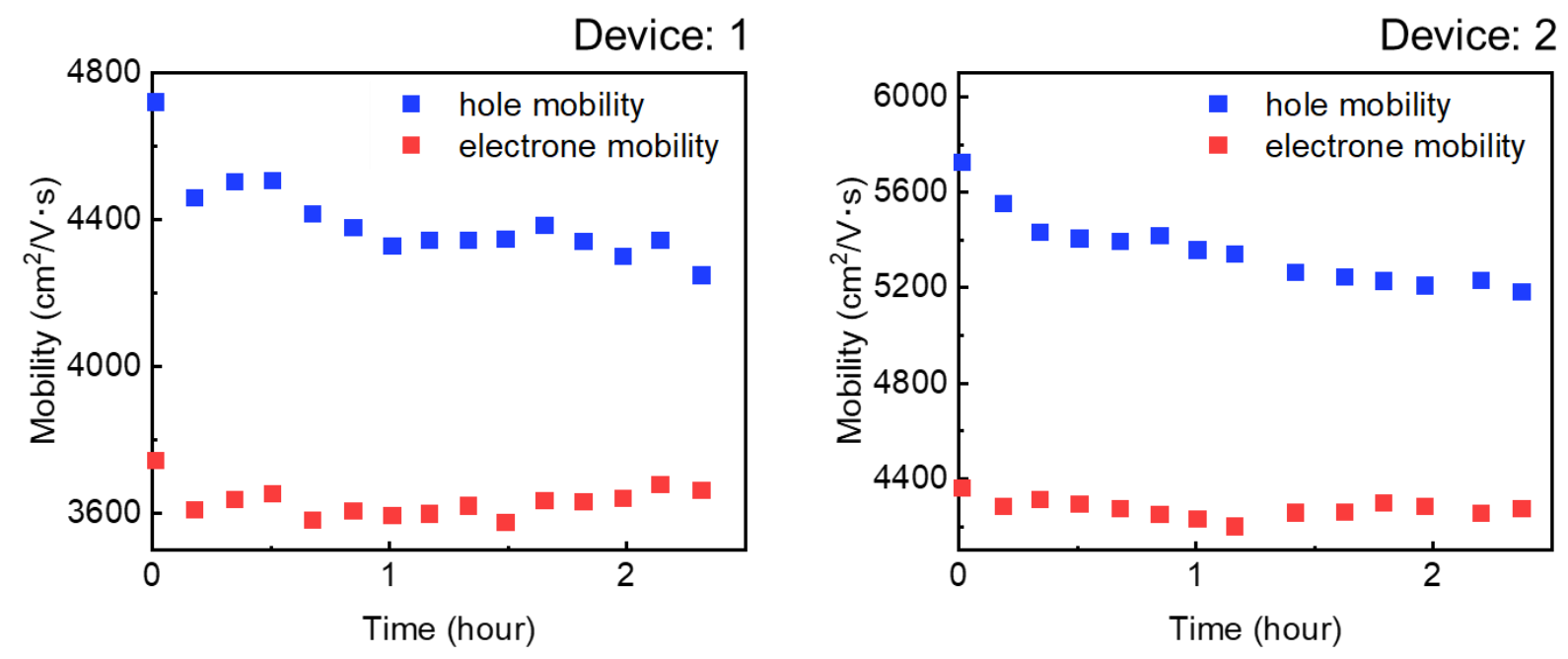

Figure S7. Time evolution of the hole (blue) and electron (red) mobility during the reaction with MeSH for the concentrations of $10 \mathrm{ppb}$ under UV irradiation. 


\section{Supporting references}

(1) Romero, F. J.; Gerardo, D.; Romero, R.; Ortiz-Gomez, I.; Salinas-Castillo, A.; MorailaMartinez, C. L.; Rodriguez, N.; Morales, D. P. Comparison of Laser-Synthetized Nanographene-Based Electrodes for Flexible Supercapacitors. Micromachines 2020, 11 (6), 555. https://doi.org/10.3390/mil1060555.

(2) Dias, G. M. V.; Müller, D.; Wesling, B. N.; Bernardes, J. C.; Hotza, D.; Rambo, C. R. Enhancing Specific Capacitance and Cyclic Stability through Incorporation of $\mathrm{MnO} 2$ into Bacterial Nanocellulose/PPy·CuCl2 Flexible Electrodes. Energy Technol. 2019, 7 (9), 1900328. https://doi.org/10.1002/ente.201900328.

(3) López, M.; Frieiro, J. L.; Nuez-Martínez, M.; Pedemonte, M.; Palacio, F.; Teixidor, F. Nanostructure ITO and Get More of It. Better Performance at Lower Cost. Nanomaterials 2020, 10 (10), 1974. https://doi.org/10.3390/nano10101974. 